\title{
Secure Information Flow by Model Checking Pushdown System*
}

\author{
Cong Sun, Liyong Tang, and Zhong Chen \\ \{suncong, tly, chen\}@infosec.pku.edu.cn \\ 1 Institute of Software, School of EECS, Peking University, China \\ 2 Key Laboratory of High Confidence Software Technologies, Ministry of Education, \\ China
}

\begin{abstract}
We propose an approach on model checking information flow for imperative language with procedures. We characterize our model with pushdown system, which has a stack of unbounded length that naturally models the execution of procedural programs. Because the type-based static analysis is sometimes too conservative and rejects safe program as ill-typed, we take a semantic-based approach by self-composing symbolic pushdown system and specifying noninterference with LTL formula. Then we verify this LTL-expressed property via model checker Moped. Except for overcoming the conservative characteristic of typebased approach, our motivation also includes the insufficient state of arts on precise information flow analysis under inter-procedural setting. To remedy the inefficiency of model checking compared with type system, we propose both compact form and contracted form of self-composition. According to our experimental results, they can greatly increase the efficiency of realistic verification. Our method provides flexibility on separating program abstraction from noninterference verification, thus could be expected to use on different programming languages.
\end{abstract}

\section{Introduction}

Noninterference is a standard criterion to formalize secure information flow. This property was first introduced by Goguen and Meseguer[1] for multi-level computing system and applied to programming language via semantic model. It commonly means any two runs of program starting in two indistinguishable states yield two indistinguishable final states. That is to say, for any pair of runs the difference on secret input are unobservable via public output.

Typical information flow analyses are studied using security type system [2][3][4][5][6]. These type systems guarantee well-typed programs do not leak any secret information. But these type-based approaches are considered overly conservative and sometimes reject safe programs, such as $l:=h \cdot 0$. Take the following program as another example,

^ This work was partially supported by the National Natural Science Foundation of China under Grant No. 60773163. 


$$
\begin{aligned}
& \text { if (l) } \mathrm{y}:=\mathrm{h} ; \text { else skip; } \\
& \text { if (! l } \mathrm{x}:=\mathrm{y} ; \text { else skip; }
\end{aligned}
$$

Suppose $l$ and $x$ are low, while $h$ and $y$ are high, standard type system[2] rejects this program over-restrictively by reporting a flow from $h$ to $x$.

To remedy this problem, semantic-based approaches[7][8] are developed, especially a recent popular approach, self-composition [9][10], which composes the original program with a variable-renamed copy to avoid considering correlative executions as pair. Model checking is an important method to verify properties of semantic-based program model. It is well-known as fully-automated and mature tool-supported. Though argued against the high complexity, model checking has been used to check noninterference, combined with abstract interpretation[11], self-composition[10][12], and type-based approach[12].

Lots of existing work get into the spectrum of discussing noninterference over more complex program constructs. Procedure is considered as a common feature of realistic imperative languages. In some applications we need to verify code from different sources together. Also there may be context-sensitive procedure calls in program. Noninterference verification of these programs involves interprocedural information flow. Consider the program below

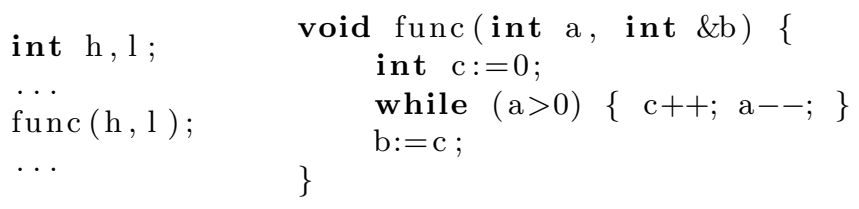

after func returns, the high variable $h$ has indirectly passed to the low variable $l$. Suppose the global variables are only observable after func returns, interprocedural analysis is required. Volpano and Smith[3] has developed a nonstandard type system to check information flow of an imperative language with procedures. But for the more precise approaches[12][10][11], procedure is not considered.

Some work treats information flow with program logic in procedural setting. Beringer et al.[13] adapt type-based approach to Hoare-like logic and introduce an auxiliary binary formulae to encode noninterference according to the principle of self-composition. Their procedure model is only restricted to parameterless form. Amtoft et al.[14] propose a Hoare-like logic to analyze inter-procedural information flow with independence assertion for object-oriented language. Method call in object-oriented language introduces additional impreciseness because of pointer aliasing on concrete location. Their procedure form is different from procedure of imperative languages and the memory model is much more complex.

Hammer et al.[15] investigate an approach for information flow control based on a well-known program slicing technique, program dependence graph (PDG). Inter-procedural information flow control can be achieved by computing summary graph and constructing system dependence graph (SDG). They introduce path condition[16][17] to improve the preciseness. Since PDG has been applied 
to handle realistic programs in C and Java, their approach seems quite general. But in their approach impreciseness comes from the construction of PDGs/SDGs. Programs should be translated into static single form (SSA) and the size of path condition should be reduced. This method is also much more expensive than type-based approaches and conservative because we cannot exclude safe program if some $\mathrm{PC}(\mathrm{y}, \mathrm{x}) \equiv$ true.

The relative insufficient study on inter-procedural information flow in state of arts motivates us to propose a general inter-procedural framework for more precise information flow analyses than the type-based approaches. Our model is based on symbolic pushdown system[18]. A pushdown system is a transition system with a stack of unbounded length in states. This unbounded stack provides a natural way to model the execution of program with procedure. The state of pushdown system includes control locations and stack symbols. Control locations are used to store global variables. Stack symbols contain both control points and local variables of procedure. This semantic model has been proved sound to our language and can be directly expressed as the input of model checker Moped[19].

The verification process consists of the following phases. First a symbolic pushdown system is derived from core-language program. Then we self-compose the derived symbolic pushdown system and express noninterference with LTL formula as the inputs of Moped. Verification is then performed by Moped. If noninterference is violated by the program model, we can get a single counterexample. This witness trace can help us find out which high variable causes the insecure information flow, thus be useful for secure program development. We have experimentally proved that our method is more precise than ordinary type-based analysis on inter-procedural information flow. Considering the relative high complexity of model checking, we have developed two derived forms of ordinary self-composition, called compact self-composition and contracted selfcomposition. The experiments indicate great efficiency improvement with these derived forms compared with ordinary self-composition. Another advantage of our approach is the flexibility unveiled by self-composing symbolic pushdown system instead of high-level language. Unlike other work did self-composition directly on high-level languages[12][10], our approach separates the possible abstractions of high-level languages from noninterference verification. Although we use a simple imperative language with procedure for simplicity, we believe it possible to apply our approach to different high-level programming languages by abstracting them into pushdown systems.

The rest of our paper is structured as follows. Section 2 sketches the simple imperative language and presents symbolic pushdown system derivation. Section 3 proposes the algorithms of both ordinary form and derived forms of selfcomposition for composing pushdown system, and introduces how to specify noninterference with LTL. We report our experiments in Section 4 and possible discussions in Section 5. 


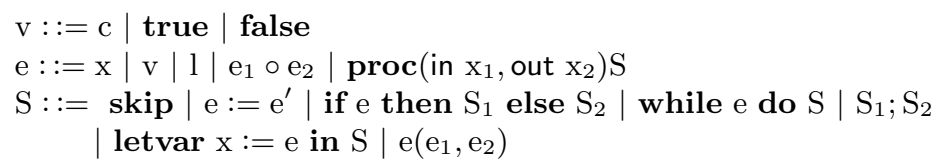

Fig. 1. Language Syntax

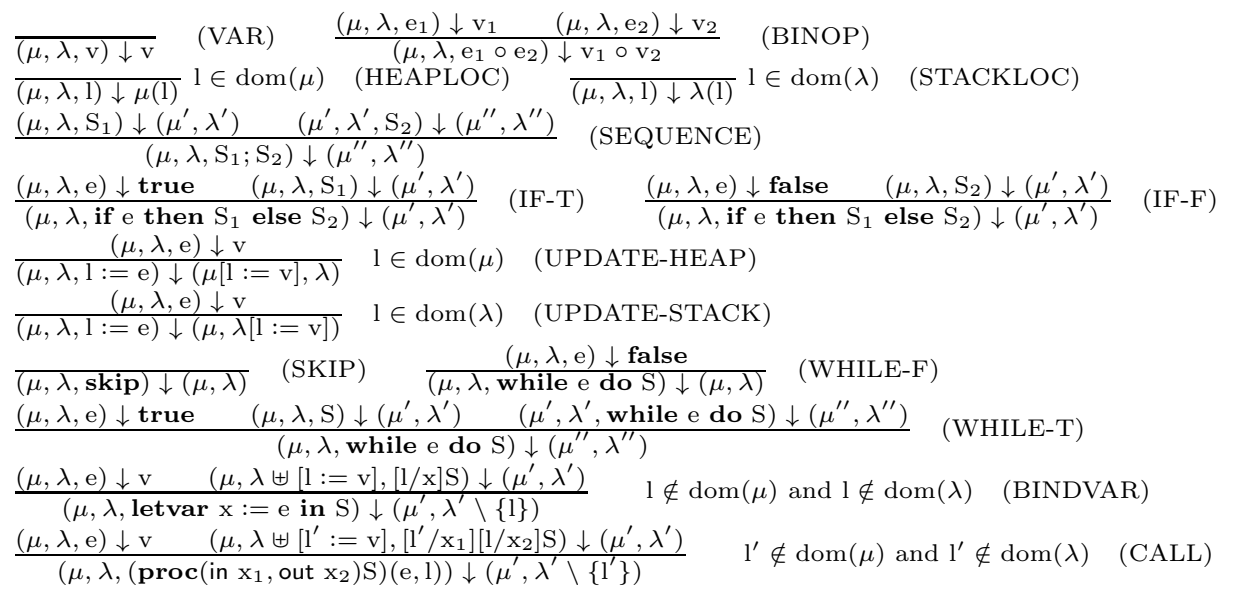

Fig. 2. Induction Rules

\section{Program Modeling}

\subsection{Language Syntax and Semantics}

The syntax of our core language with first-order procedure is given in Fig. $1 . l$ is memory location. The in parameter is local while the out parameter represents reference to global variable. The big-step operational semantics are presented in Fig. 2. $\mu, \lambda \subseteq \mathbb{L} \times \mathbb{V}$ are heap and stack respectively, where $\mathbb{L}$ is the domain of memory location and $\mathbb{V}$ is the domain of value. $\operatorname{dom}(\mu) \cap \operatorname{dom}(\lambda)=\emptyset . \lambda \uplus[l:=v]$ extends $\lambda$ with a new $l$ assigned with $v$. BINDVAR stores $v$ to new location $l$ of $\lambda$ and refers each free occurrence of $x$ in $S$ to $l$. Therefore the scope of $x$ is $S$, and $l$ is deallocated after the execution of $S$. We assume all substitutions capture-avoiding and procedure main non-recursive.

\section{$2.2 \quad$ Pushdown System}

Definition 1 (Pushdown System). A pushdown system is a triple $\mathcal{P}=$ $(P, \Gamma, \Delta) . P$ is a finite set of control location, $\Gamma$ is stack alphabet, and $\Delta \subseteq$ $(P \times \Gamma) \times\left(P \times \Gamma^{*}\right)$ is a finite set of pushdown rules. The pushdown rule has a form of $\langle p, \gamma\rangle \hookrightarrow\left\langle p^{\prime}, w\right\rangle$ to represent the relation $\left((p, \gamma),\left(p^{\prime}, w\right)\right)$, where $p, p^{\prime} \in P, \gamma \in \Gamma, w \in \Gamma^{*}$. A configuration of $\mathcal{P}$ is a pair $\langle p, w\rangle$, where $p \in P$ and $w \in \Gamma^{*}$. $w$ stands for a snapshot of stack content. Suppose the configuration set of $\mathcal{P}$ is denoted by $\operatorname{Conf}(\mathcal{P})$. We have a transition relation $\rightarrow \subseteq$ 
$\operatorname{Conf}(\mathcal{P}) \times \operatorname{Conf}(\mathcal{P})$ defined by the set of pushdown rules $\Delta$ as follows: If $\langle p, \gamma\rangle \hookrightarrow\left\langle p^{\prime}, w\right\rangle$, then $\left\langle p, \gamma w^{\prime}\right\rangle \rightarrow\left\langle p^{\prime}, w w^{\prime}\right\rangle$ for all $w^{\prime} \in \Gamma^{*}$. Let $c_{0}$ be the initial configuration. We have a transition system corresponding to the pushdown system as $\mathcal{I}_{\mathcal{P}}=\left(\operatorname{Conf}(\mathcal{P}), \rightarrow, c_{0}\right)$.

Each pushdown rule gives a pattern of program execution step. Without loss of generality, we assume $|w| \leq 2$ in that any pushdown system can be put into a normal form $(|w| \leq 2)$ with linear size increase[18].

Definition 2 (Symbolic Pushdown System). A symbolic pushdown system is a pushdown system with form $\left(P_{0} \times G, \Gamma_{0} \times L, \Delta, C_{0}\right)$. $P_{0}$ is the set of symbolic control locations, and $\Gamma_{0}$ is stack alphabet. $G$ and $L$ are respectively the domain of the control locations and stack symbols. The pushdown rules have a form $\langle(p, g)(\gamma, l)\rangle \hookrightarrow\left\langle\left(p^{\prime}, g^{\prime}\right),\left(\gamma_{1}, l_{1}\right), \ldots,\left(\gamma_{n}, l_{n}\right)\right\rangle$, where $p, p^{\prime} \in P_{0}, \gamma, \gamma_{1}, \ldots, \gamma_{n} \in$ $\Gamma_{0}$. The corresponding symbolic form separates the symbolic part from a relation of valuations:

$$
\langle p, \gamma\rangle \hookrightarrow\left\langle p^{\prime}, \gamma_{1}, \ldots, \gamma_{n}\right\rangle \quad\left(g, l, g^{\prime}, l_{1}, \ldots, l_{n}\right) \in R
$$

where $R \subseteq(G \times L) \times\left(G \times L^{n}\right)$ is a relation. Here we can also generally suppose $n \leqslant 2 . \Delta$ is the set of symbolic pushdown rules. $C_{0}=\left(\left\{p_{0}\right\} \times G\right) \times\left(\left\{y_{0}\right\} \times L\right)$ is the set of initial configurations.

The global variables are encoded into control locations and the local variables are encoded into the stack alphabet. Since we specify the control points of each procedure with stack symbols, and the global and local variables are considered as their value in $R, P_{0} \times G$ can be simplified to $G$ and the symbolic pushdown rule is of form $\langle\gamma\rangle \hookrightarrow\left\langle\gamma_{1}, \ldots, \gamma_{n}\right\rangle,\left(g, l, g^{\prime}, l_{1}, \ldots, l_{n}\right) \in R$.

\subsection{Pushdown Rules Derivation}

Suppose local variable $x$ of procedure $p$ is uniquely denoted by $\langle p, x\rangle \in \mathbb{P} \times \mathbb{Q}$, where $\mathbb{P}$ is the domain of procedure tag and $\mathbb{Q}$ is the domain of local variable name. Fig. 3 gives static analysis $\mathrm{F}$ to get the unified local variable set $\theta \subseteq \mathbb{P} \times \mathbb{Q}$. Partial function $\eta: \mathbb{P} \rightarrow 2^{\mathbb{Q}}, \eta(\mathrm{p})=\{\mathrm{x} \mid\langle\mathrm{p}, \mathrm{x}\rangle \in \theta\}$, derives all local variables of a procedure. Let $\mathbb{N}=\left\{n_{i} \mid i \in N\right\}$ be a pool of control points from which we get unique control point during pushdown rule derivation. In order to derive pushdown rules for program, we define $\Phi\left(\mathrm{S}, \mathrm{n}_{\mathrm{i}}, \mathrm{n}_{\mathrm{j}}, \mathrm{p}, \mathrm{R}\right)$ in Fig. $4 . n_{i}$ and $n_{j}$ are respectively the entry and exit control point of $S . \rho=\operatorname{dom}(\mu) . R$ is a set collecting information about variable variation and variable evaluation in

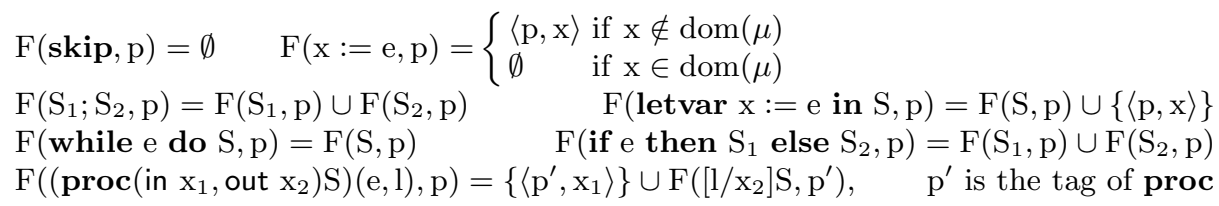

Fig. 3. Unifying Local Variables 


$$
\begin{aligned}
& \Phi\left(\text { skip, } \mathrm{n}_{\mathrm{i}}, \mathrm{n}_{\mathrm{j}}, \mathrm{p}, \mathrm{R}\right)=\left\{\left\langle\left(\rho_{\mathrm{i}}\right)\left(\mathrm{n}_{\mathrm{i}}, \eta_{\mathrm{i}}(\mathrm{p})\right)\right\rangle \hookrightarrow\left\langle\left(\rho_{\mathrm{i}}\right)\left(\mathrm{n}_{\mathrm{j}}, \eta_{\mathrm{i}}(\mathrm{p})\right)\right\rangle \mid \mathrm{R}\right\} \\
& \Phi\left(\mathrm{x}:=\mathrm{e}, \mathrm{n}_{\mathrm{i}}, \mathrm{n}_{\mathrm{j}}, \mathrm{p}, \mathrm{R}\right)=\left\{\left\langle\left(\rho_{\mathrm{i}}\right)\left(\mathrm{n}_{\mathrm{i}}, \eta_{\mathrm{i}}(\mathrm{p})\right)\right\rangle \hookrightarrow\left\langle\left(\rho_{\mathrm{j}}\right)\left(\mathrm{n}_{\mathrm{j}}, \eta_{\mathrm{j}}(\mathrm{p})\right)\right\rangle \mid \mathrm{R}^{\prime}\right\} \\
& \left\{\mathrm{R}^{\prime}=\mathrm{R} \cup\left\{\rho_{\mathrm{j}}[\mathrm{x}]=\mathrm{e}\right\} \quad \wedge \rho_{\mathrm{j}}=\rho_{\mathrm{i}}[\mathrm{x}:=\mathrm{e}] \wedge \eta_{\mathrm{j}}(\mathrm{p})=\eta_{\mathrm{i}}(\mathrm{p}), \quad \text { if } \mathrm{x} \in \rho\right. \\
& \left\{\mathrm{R}^{\prime}=\mathrm{R} \cup\left\{\eta_{\mathrm{j}}(\mathrm{p})[\mathrm{x}]=\mathrm{e}\right\} \wedge \rho_{\mathrm{j}}=\rho_{\mathrm{i}} \quad \wedge \eta_{\mathrm{j}}(\mathrm{p})=\eta_{\mathrm{i}}(\mathrm{p})[\mathrm{x}:=\mathrm{e}], \text { if } \mathrm{x} \in \eta(\mathrm{p})\right. \\
& \Phi\left(\mathrm{S}_{1} ; \mathrm{S}_{2}, \mathrm{n}_{\mathrm{i}}, \mathrm{n}_{\mathrm{j}}, \mathrm{p}, \mathrm{R}\right)=\Phi\left(\mathrm{S}_{1}, \mathrm{n}_{\mathrm{i}}, \mathrm{n}_{\mathrm{k}}, \mathrm{p}, \mathrm{R}\right) \cup \Phi\left(\mathrm{S}_{2}, \mathrm{n}_{\mathrm{k}}, \mathrm{n}_{\mathrm{j}}, \mathrm{p}, \mathrm{R}\right) \\
& \Phi\left(\text { if e then } \mathrm{S}_{1} \text { else } \mathrm{S}_{2}, \mathrm{n}_{\mathrm{i}}, \mathrm{n}_{\mathrm{j}}, \mathrm{p}, \mathrm{R}\right)=\left\{\left\langle\left(\rho_{\mathrm{i}}\right)\left(\mathrm{n}_{\mathrm{i}}, \eta_{\mathrm{i}}(\mathrm{p})\right)\right\rangle \hookrightarrow\left\langle\left(\rho_{\mathrm{i}}\right)\left(\mathrm{n}_{\mathrm{k}}, \eta_{\mathrm{i}}(\mathrm{p})\right)\right\rangle \mid \mathrm{R} \cup\{\mathrm{e}\}\right\} \\
& \cup\left\{\left\langle\left(\rho_{\mathrm{i}}\right)\left(\mathrm{n}_{\mathrm{i}}, \eta_{\mathrm{i}}(\mathrm{p})\right)\right\rangle \hookrightarrow\left\langle\left(\rho_{\mathrm{i}}\right)\left(\mathrm{n}_{\mathrm{l}}, \eta_{\mathrm{i}}(\mathrm{p})\right)\right\rangle \mid \mathrm{R} \cup\{! \mathrm{e}\}\right\} \\
& \cup \Phi\left(\mathrm{S}_{1}, \mathrm{n}_{\mathrm{k}}, \mathrm{n}_{\mathrm{j}}, \mathrm{p}, \mathrm{R} \cup\{\mathrm{e}\}\right) \cup \Phi\left(\mathrm{S}_{2}, \mathrm{n}_{\mathrm{l}}, \mathrm{n}_{\mathrm{j}}, \mathrm{p}, \mathrm{R} \cup\{! \mathrm{e}\}\right) \\
& \left\{\left\langle\left(\rho_{\mathrm{i}}\right)\left(\mathrm{n}_{\mathrm{i}}, \eta_{\mathrm{i}}(\mathrm{p})\right)\right\rangle \hookrightarrow\left\langle\left(\rho_{\mathrm{i}}\right)\left(\mathrm{n}_{\mathrm{q}}, \eta_{\mathrm{i}}(\mathrm{p})\right)\right\rangle \mid \mathrm{R} \cup\{\mathrm{e}\}\right\} \cup \Phi\left(\mathrm{S}, \mathrm{n}_{\mathrm{q}}, \mathrm{n}_{\mathrm{i}}, \mathrm{p}, \mathrm{R} \cup\{\mathrm{e}\}\right) \quad(\mathrm{P}-\mathrm{LOOP}) \\
& \Phi\left(\text { letvar } \mathrm{x}:=\mathrm{e} \text { in } \mathrm{S}, \mathrm{n}_{\mathrm{i}}, \mathrm{n}_{\mathrm{j}}, \mathrm{p}, \mathrm{R}\right)= \\
& \left\{\left\langle\left(\rho_{\mathrm{i}}\right)\left(\mathrm{n}_{\mathrm{i}}, \eta_{\mathrm{i}}(\mathrm{p})\right)\right\rangle \hookrightarrow\left\langle\left(\rho_{\mathrm{i}}\right)\left(\mathrm{n}_{\mathrm{k}}, \eta_{\mathrm{i}}(\mathrm{p})[\mathrm{x}:=\mathrm{e}]\right)\right\rangle \mid \mathrm{R} \cup\left\{\eta_{\mathrm{k}}(\mathrm{p})[\mathrm{x}]=\mathrm{e}\right\}\right\} \cup \Phi\left(\mathrm{S}, \mathrm{n}_{\mathrm{k}}, \mathrm{n}_{\mathrm{j}}, \mathrm{p}, \mathrm{R}\right) \\
& \left\{\left\langle\left(\rho_{\mathrm{i}}\right)\left(\mathrm{n}_{\mathrm{i}}, \eta_{\mathrm{i}}(\mathrm{p})\right)\right\rangle \hookrightarrow\left\langle\left(\rho_{\mathrm{i}}\right)\left(\mathrm{n}_{\mathrm{k}}, \eta_{\mathrm{k}}\left(\mathrm{p}^{\prime}\right)\left[\mathrm{x}_{1}:=\mathrm{e}\right]\right)\left(\mathrm{n}_{\mathrm{j}}, \eta_{\mathrm{i}}(\mathrm{p})\right)\right\rangle \mid \mathrm{R} \cup\left\{\eta_{\mathrm{k}}\left(\mathrm{p}^{\prime}\right)\left[\mathrm{x}_{1}\right]=\mathrm{e}\right\}\right\}
\end{aligned}
$$

Fig. 4. Derivation Rules for Pushdown System

$$
\begin{aligned}
& \left\{\left\langle\mathrm{n}_{1}\right\rangle \hookrightarrow\left\langle\mathrm{n}_{3}, \mathrm{n}_{2}\right\rangle \mid\left\{\mathrm{h}^{\prime}=\mathrm{h}, \mathrm{l}^{\prime}=\mathrm{l}, \mathrm{x}_{1}^{\prime}=\mathrm{h}, \mathrm{c}^{\prime}=\mathrm{c}\right\}\right\} \cup\left\{\left\langle\mathrm{n}_{4}\right\rangle \hookrightarrow\langle\varepsilon\rangle \mid\left\{\mathrm{h}^{\prime}=\mathrm{h}, \mathrm{l}^{\prime}=\mathrm{l}\right\}\right\} \cup \\
& \left\{\left\langle\mathrm{n}_{3}\right\rangle \hookrightarrow\left\langle\mathrm{n}_{5}\right\rangle \mid\left\{\mathrm{h}^{\prime}=\mathrm{h}, \mathrm{l}^{\prime}=\mathrm{l}, \mathrm{x}_{1}^{\prime}=\mathrm{x}_{1}, \mathrm{c}^{\prime}=0\right\}\right\} \cup \\
& \left\{\left\langle\mathrm{n}_{5}\right\rangle \hookrightarrow\left\langle\mathrm{n}_{7}\right\rangle \mid\left\{\mathrm{x}_{1}>0, \mathrm{~h}^{\prime}=\mathrm{h}, \mathrm{l}^{\prime}=1, \mathrm{x}_{1}^{\prime}=\mathrm{x}_{1}, \mathrm{c}^{\prime}=\mathrm{c}\right\}\right\} \cup \\
& \left\{\left\langle\mathrm{n}_{7}\right\rangle \hookrightarrow\left\langle\mathrm{n}_{8}\right\rangle \mid\left\{\mathrm{x}_{1}>0, \mathrm{~h}^{\prime}=\mathrm{h}, \mathrm{l}^{\prime}=1, \mathrm{x}_{1}^{\prime}=\mathrm{x}_{1}, \mathrm{c}^{\prime}=\mathrm{c}+1\right\}\right\} \cup \\
& \left\{\left\langle\mathrm{n}_{8}\right\rangle \hookrightarrow\left\langle\mathrm{n}_{5}\right\rangle \mid\left\{\mathrm{x}_{1}>0, \mathrm{~h}^{\prime}=\mathrm{h}, \mathrm{l}^{\prime}=\mathrm{l}, \mathrm{x}_{1}^{\prime}=\mathrm{x}_{1}-1, \mathrm{c}^{\prime}=\mathrm{c}\right\}\right\} \cup \\
& \left\{\left\langle\mathrm{n}_{5}\right\rangle \hookrightarrow\left\langle\mathrm{n}_{6}\right\rangle \mid\left\{\mathrm{x}_{1} \leq 0, \mathrm{~h}^{\prime}=\mathrm{h}, \mathrm{l}^{\prime}=1, \mathrm{x}_{1}^{\prime}=\mathrm{x}_{1}, \mathrm{c}^{\prime}=\mathrm{c}\right\}\right\} \cup \\
& \left\{\left\langle\mathrm{n}_{6}\right\rangle \hookrightarrow\left\langle\mathrm{n}_{4}\right\rangle \mid\left\{\mathrm{h}^{\prime}=\mathrm{h}, \mathrm{l}^{\prime}=\mathrm{c}, \mathrm{x}_{1}^{\prime}=\mathrm{x}_{1}, \mathrm{c}^{\prime}=\mathrm{c}\right\}\right\} \cup\left\{\left\langle\mathrm{n}_{2}\right\rangle \hookrightarrow\langle\varepsilon\rangle \mid\left\{\mathrm{h}^{\prime}=\mathrm{h}, \mathrm{l}^{\prime}=1\right\}\right\}
\end{aligned}
$$

Fig. 5. Symbolic Pushdown Rules

statement transition for each pushdown rule. We extend $R$ in P-BRANCH and P-LOOP to record the precondition of control flow. $R$ is initialized with $\emptyset$. $\varepsilon$ denotes the empty stack symbol.

In order to translate the derived pushdown rules into symbolic form, we suppose the control point as explicit stack symbol. We prime left-side variables of evaluation relations in $R$. Then we prime the post-transition global variables and local variables in top stack symbol, also double-prime the post-transition local variables in bottom stack symbol. For the variable holding its value during transition, extend $R$ with an equivalence relation of that variable. Then we derive the symbolic form $\left\{\left\langle n_{s}\right\rangle \hookrightarrow\left\langle\left(n_{t_{1}}\right) \ldots\left(n_{t_{k}}\right)\right\rangle \mid R\right\},(k=0,1,2)$. Fig. 5 gives the symbolic pushdown rules of the example calling procedure func in Section 1.

The derived symbolic pushdown system is sound on enforcing noninterference for the core language programs as shown in the following theorem. Let $\mu=\langle\rho, \theta\rangle$ be the state of $\mathcal{P}$. $\llbracket \mathcal{P} \rrbracket \mu$ means an execution of $\mathcal{P}$ with initial state $\mu$. It returns a final state $\mu^{\prime}$ or $\perp$ if it does not terminate. 
Theorem 1 (Soundness). Suppose $\mathcal{P}=(P, \Gamma, \Delta)$ is the pushdown system of statement $S . \Delta$ is derived by $\Phi\left(S, n_{i}, n_{j}, p_{k}, \emptyset\right) . \forall \rho_{0}, \theta_{0}, \mu_{0}, \lambda_{0}, \llbracket \mathcal{P} \rrbracket\left(\left\langle\rho_{0}, \theta_{0}\right\rangle\right)=$ $\left\langle\rho^{\prime}, \theta^{\prime}\right\rangle,\left(\mu_{0}, \lambda_{0}, S\right) \downarrow\left(\mu^{\prime}, \lambda^{\prime}\right)$. We have procedure $p_{k}$ on the top of procedure stack of $\lambda_{0}$ and $\lambda^{\prime}$. Suppose $\forall l \in \operatorname{dom}(\mu), \mu_{0}(l)=\rho_{0}[l]$, and $\forall l \in \operatorname{dom}\left(\lambda_{0}\right)$ corresponds to variable $x$ of $p_{k}, \lambda_{0}(l)=\eta_{0}\left(p_{k}\right)[x]$, we have

$\forall l \in \operatorname{dom}(\mu), \mu^{\prime}(l)=\rho^{\prime}[l]$, and $\forall l \in \operatorname{dom}\left(\lambda^{\prime}\right)$, if $l$ corresponds to variable $x$ of $p_{k}, \lambda^{\prime}(l)=\eta^{\prime}\left(p_{k}\right)[x]$.

Proof: (See Appendix for details).

\section{Noninterference Property Specification}

In order to specify noninterference, we suppose the adversary can observe whether program terminates. Then noninterference can be classified into terminationsensitive(TS) and termination-insensitive(TI). TS requires the correlative pairing executions of program both terminate or both unterminate. TI only judges the low-equivalence of final states when both executions terminate, and does not get violated if either execution unterminates. Let $L \subseteq \mu$ be the public variables of program. We define low-equivalent relation ${ }_{L}{ }_{L}$ as $\forall x \in \rho$, if $x \in L, \rho_{1}[x]=\rho_{2}[x]$, and $\forall\langle p, y\rangle \in \theta$, if $\langle p, y\rangle \in L, \eta_{1}(p)[y]=\eta_{2}(p)[y]$. Then TS and TI are formally defined as follow.

Definition 3 (TS). A pushdown system $\mathcal{P}$ has a property of TS if it satisfies $\forall \mu_{1}, \mu_{2}, \mu_{1}={ }_{L} \mu_{2} \wedge \llbracket \mathcal{P} \rrbracket \mu_{1}=\mu_{1}^{\prime} \Rightarrow \exists \mu_{2}^{\prime}, \llbracket \mathcal{P} \rrbracket \mu_{2}=\mu_{2}^{\prime} \wedge \mu_{1}^{\prime}={ }_{L} \mu_{2}^{\prime}$.

Definition 4 (TI). A pushdown system $\mathcal{P}$ has a property of TI if it satisfies $\forall \mu_{1}, \mu_{2}, \mu_{1}={ }_{L} \mu_{2} \wedge \llbracket \mathcal{P} \rrbracket \mu_{1}=\mu_{1}^{\prime} \Rightarrow \llbracket \mathcal{P} \rrbracket \mu_{2}=\perp \vee\left(\exists \mu_{2}^{\prime}, \llbracket \mathcal{P} \rrbracket \mu_{2}=\mu_{2}^{\prime} \wedge \mu_{1}^{\prime}={ }_{L} \mu_{2}^{\prime}\right)$.

TI can be simplified by restricting the precondition:

Definition $5\left(\mathbf{T I}^{\prime}\right)$. A pushdown system $\mathcal{P}$ has a property of $\mathrm{TI}$ if it satisfies $\forall \mu_{1}, \mu_{2}, \mu_{1}={ }_{L} \mu_{2} \wedge \llbracket \mathcal{P} \rrbracket \mu_{1}=\mu_{1}^{\prime} \wedge \llbracket \mathcal{P} \rrbracket \mu_{2}=\mu_{2}^{\prime} \Rightarrow \mu_{1}^{\prime}={ }_{L} \mu_{2}^{\prime}$.

For simplicity, we only consider TI in the following. The LTL property w.r.t TS can be derived similarly by method in [9].

\subsection{Ordinary Self-Composition}

The primary motivation of self-composition is to model two correlative runs of program with indistinguishable inputs as a single run of composed program, which greatly benefits the algorithmic verification techniques, such as model checking, on the property expressing via temporal logics.

Suppose $\mathcal{P}_{1}$ and $\mathcal{P}_{2}$ are pushdown systems. $\mu_{1}, \mu_{1}^{\prime}$ are states of $\mathcal{P}_{1}$, and $\mu_{2}$, $\mu_{2}^{\prime}$ are states of $\mathcal{P}_{2}$. Let $\mu_{1} \cap \mu_{2}=\left(\rho_{1} \cap \rho_{2}\right) \cup\left(\theta_{1} \cap \theta_{2}\right)$. $\oplus$ is disjoint union of memory. If $\mu_{1} \cap \mu_{2}=\emptyset$, we define disjoint composition operation $\triangleright$ of $\mathcal{P}_{1}$ and $\mathcal{P}_{2}$ as

$\llbracket \mathcal{P}_{1} \rrbracket\left(\mu_{1} \oplus \mu\right)=\left(\mu_{1}^{\prime} \oplus \mu\right)$ for some $\mu$, and $\llbracket \mathcal{P}_{2} \rrbracket\left(\mu^{\prime} \oplus \mu_{2}\right)=\left(\mu^{\prime} \oplus \mu_{2}^{\prime}\right)$ for some $\mu^{\prime}$, iff $\llbracket \mathcal{P}_{1} \triangleright \mathcal{P}_{2} \rrbracket\left(\mu_{1} \oplus \mu_{2}\right)=\left(\mu_{1}^{\prime} \oplus \mu_{2}^{\prime}\right)$. 
Suppose $\mu=\langle\rho, \theta\rangle$ be the state of $\mathcal{P}$. The state of $\mathcal{P}[\xi]$ is derived by renaming the variables of $\mathcal{P}$ by function $\xi: \mu \rightarrow\left\langle\left\{x^{*} \mid x \in \rho\right\},\left\{\left\langle p, y^{*}\right\rangle \mid\langle p, y\rangle \in \theta\right\}\right\rangle$, such that $\forall x \in \rho, \xi(x)=x^{*}$ and $\forall\langle p, y\rangle \in \theta, \xi(\langle p, y\rangle)=\left\langle p, y^{*}\right\rangle$. The ordinary selfcomposition is defined based on disjoint composition and variable renaming, and the corresponding TI is defined as:

Definition 6 (TI, ordinary self-composition). $\mathcal{P}$ has a property of TI iff

$$
\mu_{1}={ }_{L} \mu_{2} \wedge \llbracket \mathcal{P} \triangleright \mathcal{P}[\xi] \rrbracket\left(\mu_{1} \oplus \mu_{2}\right)=\left(\mu_{1}^{\prime} \oplus \mu_{2}^{\prime}\right) \Rightarrow \mu_{1}^{\prime}={ }_{L} \mu_{2}^{\prime},
$$

where $\mu_{1}, \mu_{1}^{\prime}$ are states of $\mathcal{P}$, and $\mu_{2}, \mu_{2}^{\prime}$ are states of $\mathcal{P}[\xi]$.

The corresponding self-composing algorithm on pushdown system to derive $\mathcal{P} \triangleright \mathcal{P}[\xi]$ is proposed as follow.

1. Derive $\mathcal{P}[\xi]$ by substituting variables of $\mathcal{P}$ with the corresponding renamed variables defined by $\xi$, and substituting each control point $n_{i}$ of $\mathcal{P}$ with $n_{i}^{*}$.

2. Merge the pushdown rules of $\mathcal{P}$ and $\mathcal{P}[\xi]$.

3. Modify the last pushdown rule of $\mathcal{P}$ from $\left\langle n_{\text {final }}\right\rangle \hookrightarrow\langle\varepsilon\rangle$ to $\left\langle n_{\text {final }}\right\rangle \hookrightarrow\left\langle n_{\text {init }}^{*}\right\rangle$, and for the totalness of composed pushdown system, modify the last pushdown rule of $\mathcal{P}[\xi]$ from $\left\langle n_{\text {final }}^{*}\right\rangle \hookrightarrow\langle\varepsilon\rangle$ to $\left\langle n_{\text {final }}^{*}\right\rangle \hookrightarrow\left\langle n_{\text {final }}^{*}\right\rangle$. Algorithm 1 is used to find the last transition of original pushdown system.

4. For each rule of $\mathcal{P}, \forall x^{*} \in \rho_{2}$, add $x^{* \prime}=x^{*}$ to $R$. For each $\left\langle n_{i}\right\rangle \hookrightarrow\left\langle n_{j}\right\rangle$ of $\mathcal{P}$, $\forall\left\langle p, y^{*}\right\rangle \in \theta_{2}$, add $\eta(p)\left[y^{*}\right]^{\prime}=\eta(p)\left[y^{*}\right]$ to $R$. For each $\left\langle n_{i}\right\rangle \hookrightarrow\left\langle n_{k}, n_{j}\right\rangle$ of $\mathcal{P}$, $\forall\left\langle p, y^{*}\right\rangle \in \theta_{2}$, add $\eta(p)\left[y^{*}\right]^{\prime \prime}=\eta(p)\left[y^{*}\right]$ to $R$.

5. For each rule of $\mathcal{P}[\xi], \forall x \in \rho_{1}$, add $x^{\prime}=x$ to $R$. For each $\left\langle n_{i}^{*}\right\rangle \hookrightarrow\left\langle n_{j}^{*}\right\rangle$ of $\mathcal{P}[\xi]$, $\forall\langle p, y\rangle \in \theta_{1}$, add $\eta(p)[y]^{\prime}=\eta(p)[y]$ to $R$. For each $\left\langle n_{i}^{*}\right\rangle \hookrightarrow\left\langle n_{k}^{*}, n_{j}^{*}\right\rangle$ of $\mathcal{P}[\xi]$, $\forall\langle p, y\rangle \in \theta_{1}$, add $\eta(p)[y]^{\prime \prime}=\eta(p)[y]$ to $R$.

6. $\forall\langle p, y\rangle \in \theta_{1}$, add $\eta(p)[y]^{\prime}=\eta(p)[y]$ to $R$ of $\left\langle n_{\text {final }}\right\rangle \hookrightarrow\left\langle n_{\text {init }}^{*}\right\rangle$.

Then TI can be expressed by linear temporal logic and verified on this composed pushdown system by model checking. $\mathcal{P}$ has a property of TI if and only if $\mathcal{P} \triangleright \mathcal{P}[\xi]$ satisfies $\left(\mu_{1}={ }_{L} \mu_{2}\right) \Rightarrow \mathrm{G}\left(n_{\text {final }}^{*} \Rightarrow\left(\mu_{1}={ }_{L} \mu_{2}\right)\right)$.

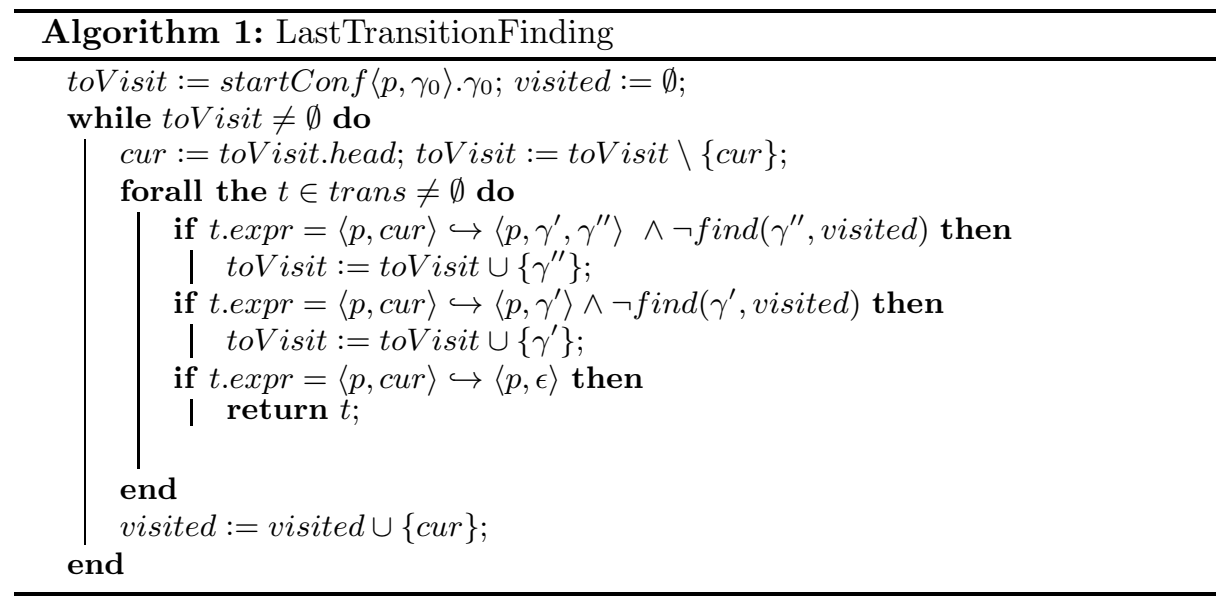




\subsection{Compact Self-Composition}

Since our approach is based on symbolic model checking, the variable count has great impact on the size of binary decision diagram (BDD) and performance of model checker. The increase in variable count after ordinary self-composition seriously decreases the efficiency of model checking. This motivates us to find new self-composing methods in inter-procedural settings. The compact form of self-composition relies on the following two assumptions

- All the variables observable by the adversary are global. That means to treat the procedure with observation point in it as main procedure.

- All local variables are initialized before being used in procedure, and vanish while procedure returns, thus are considered as high.

Let $\mu_{1} \oplus_{l} \mu_{2}=\mu_{1} \oplus \rho_{2}=\left\langle\rho_{1}, \theta_{1}\right\rangle \oplus \rho_{2}$, and $\mu_{1} \oplus_{r} \mu_{2}=\rho_{1} \oplus \mu_{2}=\rho_{1} \oplus\left\langle\rho_{2}, \theta_{2}\right\rangle$. Suppose $\oplus_{\rho}$ is the disjoint union of global variables, we have compact disjoint composition $\triangleright_{\rho}$ of $\mathcal{P}_{1}$ and $\mathcal{P}_{2}$, where $\llbracket \mathcal{P}_{1} \rrbracket\left(\mu_{1} \oplus_{l} \mu\right)=\left(\mu_{1}^{\prime} \oplus \mu\right)$ for some $\mu$, and $\llbracket \mathcal{P}_{2} \rrbracket\left(\mu^{\prime} \oplus_{r} \mu_{2}\right)=\left(\mu^{\prime} \oplus \mu_{2}^{\prime}\right)$ for some $\mu^{\prime}$, iff $\llbracket \mathcal{P}_{1} \triangleright_{\rho} \mathcal{P}_{2} \rrbracket\left(\mu_{1} \oplus_{\rho} \mu_{2}\right)=\left(\mu_{1}^{\prime} \oplus_{\rho} \mu_{2}^{\prime}\right)$.

Definition 7 (TI,compact self-composition). $\mathcal{P}$ has a property of TI iff

$$
\rho_{1}={ }_{L} \rho_{2} \wedge \llbracket \mathcal{P} \triangleright_{\rho} \mathcal{P}[\xi] \rrbracket\left(\mu_{1} \oplus_{\rho} \mu_{2}\right)=\left(\mu_{1}^{\prime} \oplus_{\rho} \mu_{2}^{\prime}\right) \Rightarrow \rho_{1}^{\prime}=_{L} \rho_{2}^{\prime}
$$

The self-composing algorithm w.r.t compact form is derived by modifying step 4 to step 6 of the algorithm in Section 3.1 to the following strategies

$4^{\prime}$. For each rule of $\mathcal{P}, \forall x^{*} \in \rho_{2}$, add $x^{* \prime}=x^{*}$ to $R$.

$5^{\prime}$. For each rule of $\mathcal{P}[\xi], \forall x \in \rho_{1}$, add $x^{\prime}=x$ to $R$.

A unified algorithm of ordinary and compact forms of self-composition is illustrated by Algorithm 2. Suppose $\mathcal{P}$.lvars : $C P \mapsto 2^{\mathbb{Q}}$ be a mapping from the set of control points to a set of local variables related to certain procedure. Also suppose procedure tag $p_{i}$ is represented by the set of control points arrived during execution of this procedure. Then we have $\mathcal{P}$.lvars $(\gamma)=\left\{x \mid \gamma \in p_{i},\left\langle p_{i}, x\right\rangle \in \theta\right\}$.

\subsection{Contracted Self-Composition}

According to the assumptions given in Section 3.2, we know that noninterference property at certain observation point of program does not really care the value of local variables in the callee procedures. By the algorithm of Section 3.2, we have reduced the length of $R$ accompanying pushdown rules. But what if we want to reduce the states of composed program? Since the adversary can only observe the global variables of main procedure, we can avoid duplicating memory of local variables by making the composed part of main procedure call the original callee, instead of the composed callee, as original part of main procedure does. This form of self-composition additionally relies on the following assumption:

- Global variable can not be used in callee procedure unless it is passed as parameter of callee procedure.

With the contracted form of self-composition, we do not compose callee procedures. Therefore we could not express the direct effect of newly defined global variables on local variables of callee procedures. The state of $\mathcal{P}\left[\xi^{\prime}\right]$ is derived by $\xi^{\prime}:\langle\rho, \theta\rangle \rightarrow\left\langle\left\{x^{*} \mid x \in \rho\right\}, \theta\right\rangle$ such that $\forall x \in \rho, \xi^{\prime}(x)=x^{*}$ and $\forall\langle p, y\rangle \in$ $\theta, \xi^{\prime}(\langle p, y\rangle)=\langle p, y\rangle$. We have TI w.r.t a contracted form. 


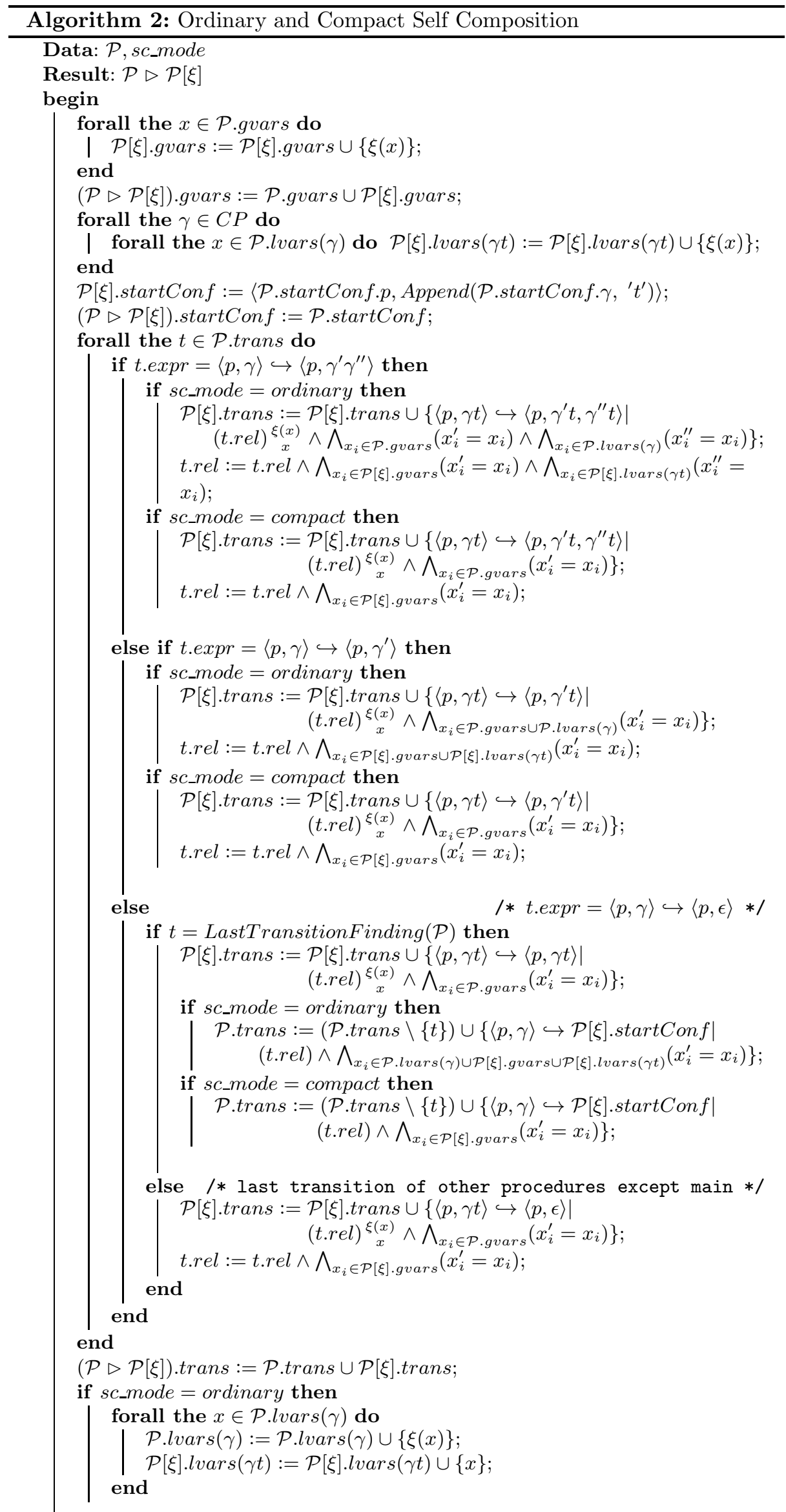


Definition 8 (TI,contracted self-composition). $\mathcal{P}$ has a property of TI iff

$$
\rho_{1}={ }_{L} \rho_{2} \wedge \llbracket \mathcal{P} \triangleright_{\rho} \mathcal{P}\left[\xi^{\prime}\right] \rrbracket\left(\mu_{1} \oplus_{\rho} \mu_{2}\right)=\left(\mu_{1}^{\prime} \oplus_{\rho} \mu_{2}^{\prime}\right) \Rightarrow \rho_{1}^{\prime}={ }_{L} \rho_{2}^{\prime}
$$

The related self-composing algorithm distinguishes pushdown rules of main procedure from pushdown rules of callee procedures.

1. Derive $\mathcal{P}\left[\xi^{\prime}\right]$ by substituting variables of $\mathcal{P}$ with renamed variables defined by $\xi^{\prime}$. For pushdown rules of main procedure, substitute each $\left\langle n_{i}\right\rangle \hookrightarrow\left\langle n_{j}\right\rangle$ with $\left\langle n_{i}^{*}\right\rangle \hookrightarrow\left\langle n_{j}^{*}\right\rangle,\left\langle n_{i}\right\rangle \hookrightarrow\langle\varepsilon\rangle$ with $\left\langle n_{i}^{*}\right\rangle \hookrightarrow\langle\varepsilon\rangle$, and $\left\langle n_{i}\right\rangle \hookrightarrow\left\langle n_{k}, n_{j}\right\rangle$ with $\left\langle n_{i}^{*}\right\rangle \hookrightarrow\left\langle n_{k}, n_{j}^{*}\right\rangle$.

2. Merge $\mathcal{P}\left[\xi^{\prime}\right]$ and $\mathcal{P}$, taking duplicated pushdown rules only once.

3. Same as step 3 in Section 3.1.

4. Same as step $4^{\prime}$ and $5^{\prime}$ in Section 3.2.

The corresponding LTL-expressed TI for compact self-composition and contracted self-composition is $\left(\rho_{1}={ }_{L} \rho_{2}\right) \Rightarrow \mathrm{G}\left(n_{\text {final }}^{*} \Rightarrow\left(\rho_{1}={ }_{L} \rho_{2}\right)\right)$. Let $R T$ be the set of global variables used as the store of return value of the callee procedures. The algorithm is described in detail by Algorithm 3. Our experiments in Section 4 illustrate the improvement on efficiency brought by these derived forms of self-composition.

\section{Experiments}

The main purpose of our experiments is to reveal that the derived forms of selfcomposition, compared with the ordinary form, can really improve the efficiency of verification. Also we make clear how we could benefit from the procedural settings and model checking.

We have implemented all three forms of self-composition for symbolic pushdown system. This is a static transformation before the pushdown system parsing phase. Self-composed pushdown system is generated and related Moped options are set. We add command-line options to Moped version 1 . We also implicitly require the derived variables and control points in composed program tagged by postfix - $t$, especially the final control point named as -final. With this assumption, user can express TI/TS noninterference by LTL from the original symbolic pushdown system instead of the one after self-composition. All experiments were performed on a laptop with $1.66 \mathrm{GHz}$ Intel Core $2 \mathrm{CPU}$ and $1 \mathrm{~GB}$ RAM running Linux Fedora Core 6. The BDD library used by Moped is CUDD 2.3.1. Experimental results are partially presented in Table 1.

In this table, \#gvars and \#lvars give the number of global variables and the number of local variables respectively. \#pubs represents the number of public/low variables. $N$ gives the number of bit of each integer variable. As shown in Fig. 6, larger $N$ causes increase in consumed time and memory. Fortunately however, the secure/insecure judgement made by our method is mostly insensitive to $N$, thus we could set $N$ as small as possible to outperform other methods. The only matter is to ensure the range of integer $\left(0 \sim 2^{N}-1\right)$ cover the constant value assigned to it. The basic checking result Yes reports the program is secure, while the answer No, along with the witness path generated as a counterexample, not only tells the program is insecure, but facilitates our method on 

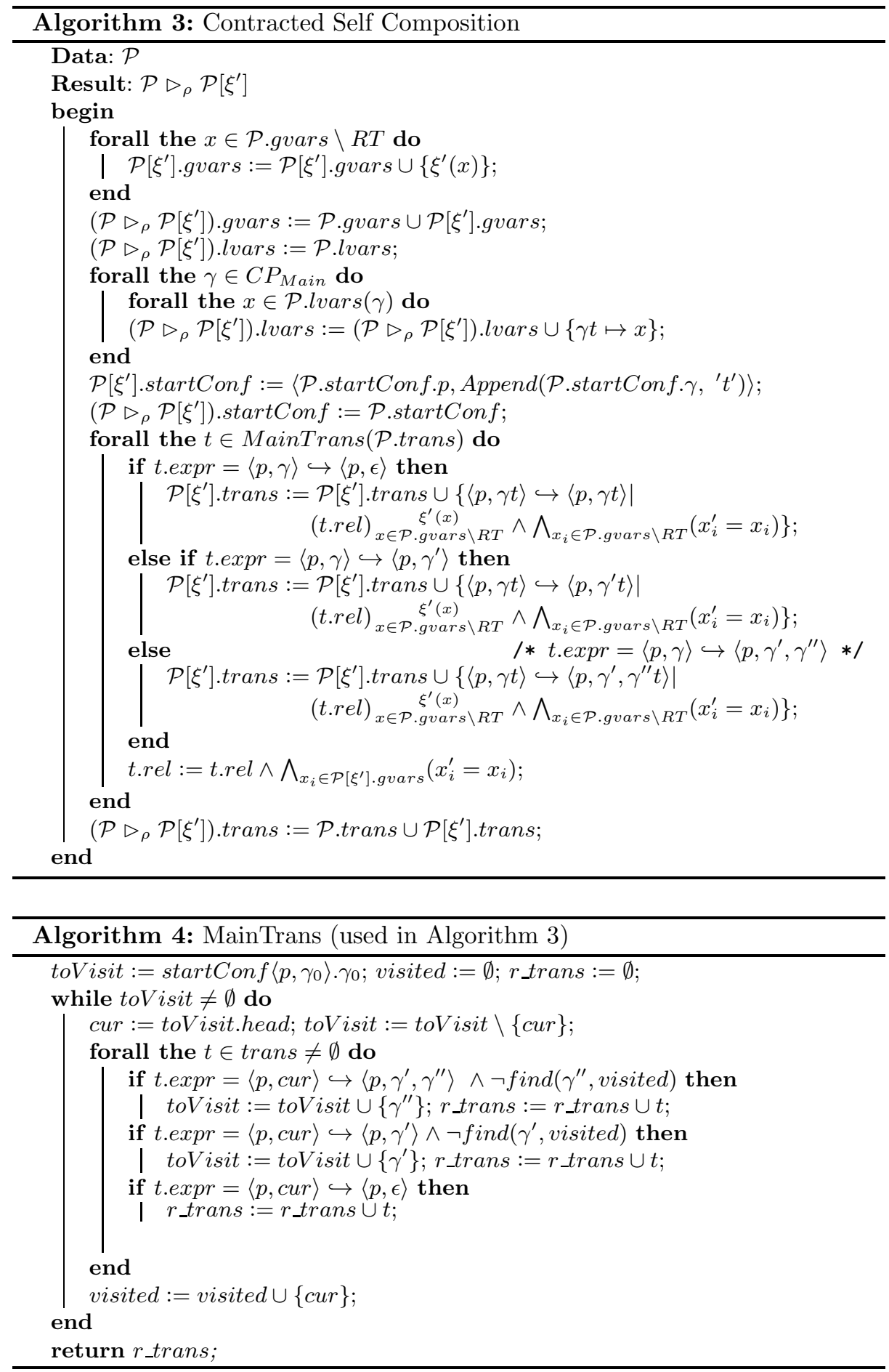


\begin{tabular}{|l||c|c|c|c|c|c||c|c|c|c|}
\hline Prog & Prog & \#gvars & \#lvars & \#pubs & Yes & N & \multicolumn{2}{|c|}{ ordinary } & \multicolumn{2}{c|}{ compact } \\
\cline { 8 - 11 } & & & & & No & & Time(s) & Mem(MB) & Time(s) & Mem(MB) \\
\hline \hline ttaa1 & 13 & 3 & 2 & 2 & Yes & 9 & 0.01 & 45.82 & 0.01 & 45.58 \\
\hline ttaa2 & 14 & 3 & 2 & 2 & No & 8 & 0.16 & 41.99 & 0.03 & 41.95 \\
\hline ttaa3 & 22 & 4 & 3 & 3 & Yes & 8 & 1.86 & 49.61 & 1.52 & 48.87 \\
\cline { 8 - 11 } & & & & & & 1 & $<0.01$ & 4.74 & $<0.01$ & 4.71 \\
\hline lgj & 13 & 3 & 1 & 2 & Yes & 9 & $<0.01$ & 4.65 & $<0.01$ & 4.65 \\
\hline func1 & 12 & 2 & 2 & 1 & No & 8 & 4.45 & 36.68 & 2.48 & 19.02 \\
\hline hu1 & 54 & 2 & 33 & 1 & No & 1 & - & - & 12.10 & 73.19 \\
\hline hu2 & 70 & 2 & 17 & 1 & No & 3 & 4.11 & 53.61 & 0.21 & 14.62 \\
\hline hu2_func & 26 & 3 & 1 & 1 & No & 8 & 1.44 & 35.63 & 1.40 & 34.92 \\
\hline hu3 & 63 & 60 & 0 & 20 & No & 1 & - & - & 0.07 & 202.56 \\
\hline hu3* & 63 & 60 & 0 & 40 & No & 1 & - & - & 128.16 & 1015.61 \\
\hline mod_imap & 526 & 9 & 13 & 8 & No & 4 & 14.00 & 34.55 & 11.25 & 32.88 \\
\cline { 8 - 11 } & & & & & & 5 & 404.79 & 65.40 & 327.93 & 55.09 \\
\hline
\end{tabular}

Table 1. Experimental Results

reasoning the flow path from certain high variable to low variables. In another word, the single counterexample identified by model checker could tell us the flow-source variable with security level high. This is the first step to fix the flaw of program, and probably the most obvious benefit provided by model checking. Consider again the program calling func in Section 1, the snapshot is presented in Fig. 7 where $N=1$. We can clearly observe the difference of $l$ and $l t$ at observation point comes from the difference on value of $h$ and $h t$ at the beginning of execution. The illegal flow is performed by transition rule from $\mathrm{f} 4$ to $\mathrm{f} 5$.

Program ttaa1, ttaa2 and ttaa3 are respectively programs presented in Figure 1, Figure 4 and Figure 9 of [10]. In [10] the authors report a failure on verifying ttaa3 secure using BLAST. Here using our method we can verify this program secure. hu1, hu2 and hu3 model the program expr1.c, expr2.c and expr4.c of [12]. In order to explain the benefit from procedural setting, we encapsulate the if-branch of hu2 into a procedure and change hu2 to a program calling this procedure for sixteen times. The resulting program is hu2_func. The evaluation results are illustrated by Fig. 6. Compared with ordinary selfcomposition of hu2, consumed time and memory are greatly reduced by the ordinary self-composition of hu2_func. To make clear the effectiveness of the derived forms of self-composition, we did ordinary and compact self-composition on hu2 (since no procedure exists, the contracted form is not available) and all three forms of self-composition on hu2_func. The time reduction by using compact form is more notable on hu2 than on hu2_func. This is because hu2 has more local variables, and the conjunction of relations in $R$ is much shorter for compact form than ordinary form. That means each symbolic pushdown rule of derived pushdown system corresponds to more explicit pushdown rules and smaller BDD. The only one local variable of hu2_func could not make this effect 

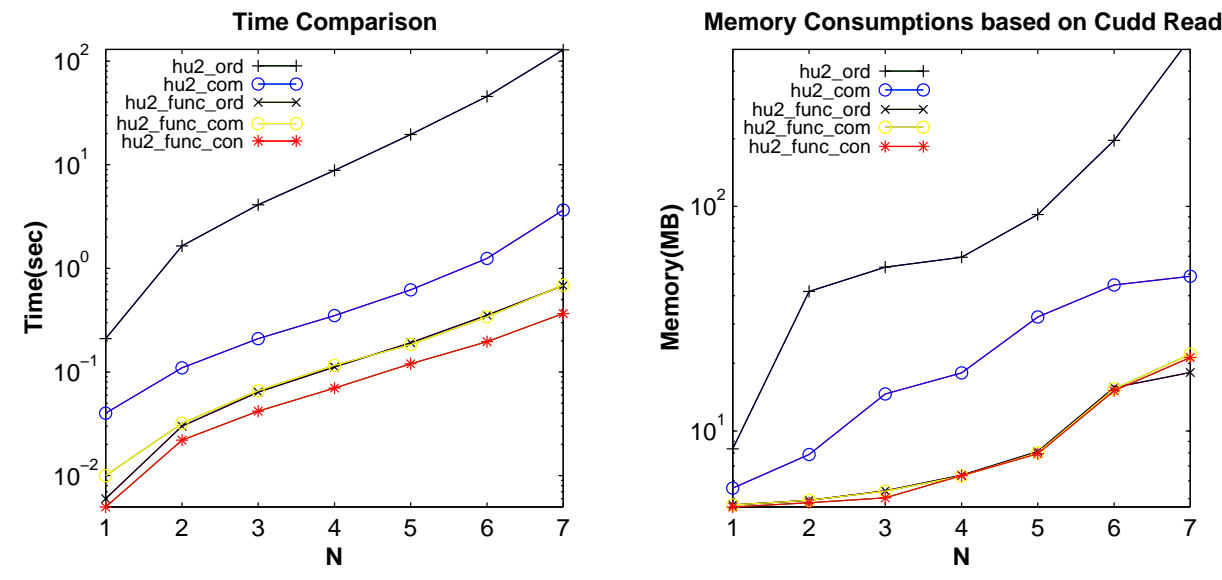

Fig. 6. Time and Memory Comparison

obvious. The contracted form could outperform the compact form because the number of derived local variables is reduced to half compared with compact self-composition and the number of pushdown rules is also reduced. hu3 can be verified insecure in two aspects. First, it can leak path information of whether branch with condition $b_{10}$ is taken. Here we need to specify the initial value of $\operatorname{low}_{\mathrm{i}}(i=1,2, \ldots, 20)$ to be 0 (See hu3 in Table 1 ). Second, we can reveal that low $_{10}$ leaks high 10 by specifying the original part and the composed part of derived pushdown system take the same path $\left(b_{i}=b t_{i}, i=1,2, \ldots, 20\right)$ (See hu3* in Table 1). Here the path conditions $b_{i}$ should be global. Without any local variable, the ordinary form and compact form of hu3 are of no difference and we record experiment results of either instead of both in Table 1.

To show the realistic usage of our method, we have verified the standard module mod_imap.c of Apache 1.3.23. This program is also verified as an important experiment in [12]. In the imap_url function of this program, a possible tainted Referer url could be returned and passed to menu_* functions and then to the arguments of ap_rvputs. ap_rvputs passes these arguments to client browser and causes a cross-site scripting (XSS) vulnerability. To check crosssite scripting, we need to consider the parameters of ap_rvputs as variables with high integrity, while the returned HTTP_REFERER has low integrity. The dual verification problem on integrity means to decide the high variables hold the following relation $\left(\mu_{1}={ }_{H} \mu_{2}\right) \Rightarrow G\left(n_{\text {final }}^{*} \Rightarrow\left(\mu_{1}={ }_{H} \mu_{2}\right)\right)$. Since Moped can only deal with integer and boolean variables and array of both types, we have to first abstract string to integer, and model the string operations using binary operations of integers. To ensure that the abstract integer variable could be assigned by constant value modeling constant string, $N$ could not be less than 4 . We have successfully verified this program insecure, and according to the experimental results, the efficiency of our method under proper abstraction is competitive to other methods [12]. 


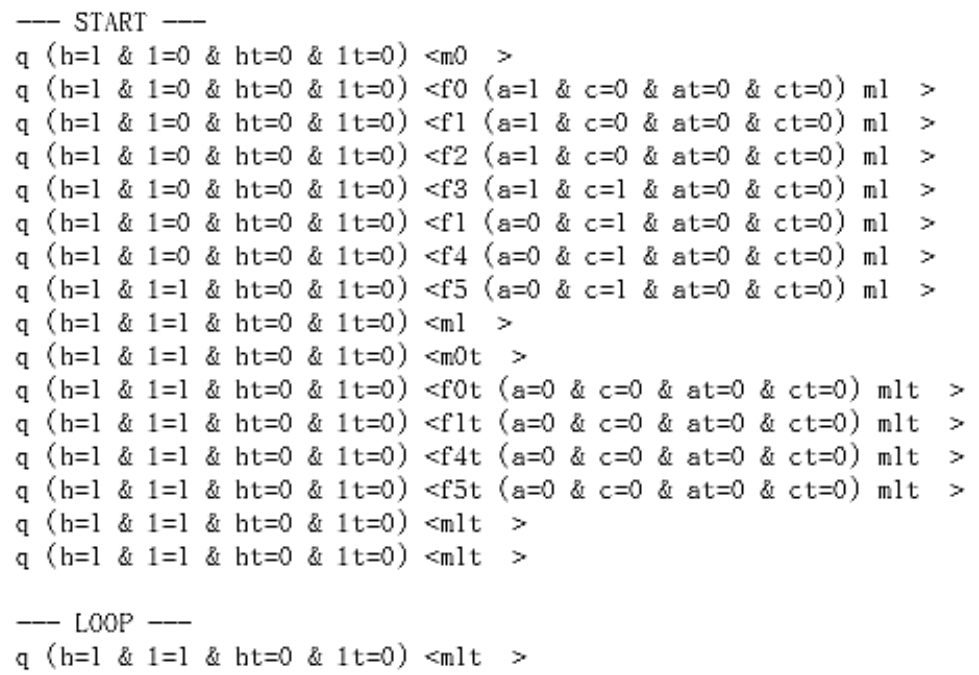

Fig. 7. Snapshot of Counterexample

\section{Discussions}

To the language presented in Section 2.1, we require the out parameter of procedure to refer to global variable. Because in symbolic pushdown rules of callee procedure, the stack symbols do not contain the stack symbol of caller. We cannot express the effect to the local variables of caller directly in one pushdown rule, including return action, of callee. This problem comes from the definition of transition relation $\rightarrow$ of $\mathcal{I}_{\mathcal{P}}$ corresponding to pushdown system $\mathcal{P}$. We can solve this problem by storing the out value referring to the caller's local variable in the common part of caller's rules and callee's rules, that is the global variables as control locations. That means we need to add an additional control location $r$ for the out value. In P-PROC of Fig. 4, we need to substitute $x_{2}$ in procedure body $\mathrm{S}$ with $\mathrm{r}$ instead of 1 , and add a rule for the caller to assign value of $r$ to its local variable. This additional global variable could also help us to store the return value of language whose procedure has side effect. A possible ambiguity is the security level of this additional global variable. We could set it high to avoid invalid information flow caused by its change. Also we do not consider parameters with inout type since an inout parameter can be trivially eliminated by one in and one out parameter.

Model checking based approaches are usually argued against the efficiency problem and state explosion. Although the derived forms of self-composition we develop could greatly improve the efficiency, the complexity of satisfiability for LTL is complete for PSPACE[20][21]. Also the ordinary partial correctness specified by self-composition is undecidable while type-based approaches are generally much faster. 
As we have stated in last section, Moped can only deal with boolean and integer variables and arrays, and each integer variable should have a finite range of $0 \sim 2^{N}-1$. This makes abstraction necessary for verifying realistic programs. So we plan to scale up our approach to more real applications by adapting proper approaches on abstraction, possibly using some recently developed tools[22][23]. Because all forms of self-composition are developed on symbolic pushdown system, the influence of abstraction on self-composition should be little.

When the program has a great many constants, certain information flow will be omitted if the initial $N$ is too small. But larger $N$ means increase on time and memory consumption, as we can see in Fig. 6. Therefore it is sometimes difficult to balance the exactness and the efficiency by choosing proper initial value of $N$. Another restriction of our approach is that with the deterministic nature of the model our approach has not scaled to concurrent programs. Our future work will adapt our approach to handle concurrency using some formal models[24][25].

\section{References}

1. Goguen, J.A., Meseguer, J.: Security policies and security models. In: IEEE Symposium on Security and Privacy. (1982) 11-20

2. Volpano, D.M., Irvine, C.E., Smith, G.: A sound type system for secure flow analysis. Journal of Computer Security 4(2/3) (1996) 167-188

3. Volpano, D.M., Smith, G.: A type-based approach to program security. In Bidoit, M., Dauchet, M., eds.: TAPSOFT. Volume 1214 of Lecture Notes in Computer Science., Springer (1997) 607-621

4. Smith, G., Volpano, D.M.: Secure information flow in a multi-threaded imperative language. In: POPL. (1998) 355-364

5. Heintze, N., Riecke, J.G.: The slam calculus: Programming with secrecy and integrity. In: POPL. (1998) 365-377

6. Sabelfeld, A., Myers, A.C.: Language-based information-flow security. IEEE Journal on Selected Areas in Communications 21(1) (2003) 5-19

7. Joshi, R., Leino, K.R.M.: A semantic approach to secure information flow. Sci. Comput. Program. 37(1-3) (2000) 113-138

8. Sabelfeld, A., Sands, D.: A per model of secure information flow in sequential programs. Higher-Order and Symbolic Computation 14(1) (2001) 59-91

9. Barthe, G., D'Argenio, P.R., Rezk, T.: Secure information flow by self-composition. In: CSFW, IEEE Computer Society (2004) 100-114

10. Terauchi, T., Aiken, A.: Secure information flow as a safety problem. In Hankin, C., Siveroni, I., eds.: SAS. Volume 3672 of Lecture Notes in Computer Science., Springer (2005) 352-367

11. Francesco, N.D., Santone, A., Tesei, L.: Abstract interpretation and model checking for checking secure information flow in concurrent systems. Fundam. Inform. 54(23) (2003) 195-211

12. Unno, H., Kobayashi, N., Yonezawa, A.: Combining type-based analysis and model checking for finding counterexamples against non-interference. In Sreedhar, V.C., Zdancewic, S., eds.: PLAS, ACM (2006) 17-26

13. Beringer, L., Hofmann, M.: Secure information flow and program logics. In: CSF, IEEE Computer Society (2007) 233-248 
14. Amtoft, T., Bandhakavi, S., Banerjee, A.: A logic for information flow in objectoriented programs. In Morrisett, J.G., Jones, S.L.P., eds.: POPL, ACM (2006) 91-102

15. Hammer, C., Krinke, J., Snelting, G.: Information flow control for java based on path conditions in dependence graphs. In: In IEEE International Symposium on Secure Software Engineering. (2006)

16. Snelting, G., Robschink, T., Krinke, J.: Efficient path conditions in dependence graphs for software safety analysis. ACM Trans. Softw. Eng. Methodol. 15(4) (2006) 410-457

17. Snelting, G.: Combining slicing and constraint solving for validation of measurement software. In Cousot, R., Schmidt, D.A., eds.: SAS. Volume 1145 of Lecture Notes in Computer Science., Springer (1996) 332-348

18. Schwoon, S.: Model Checking Pushdown Systems. PhD thesis, Technical University of Munich, Munich, Germany (2002)

19. Kiefer, S., Schwoon, S., Suwimonteerabuth, D.: Moped: A model-checker for pushdown systems (2002) http://www.fmi.uni-stuttgart.de/szs/tools/moped/.

20. Sistla, A.P., Clarke, E.M.: The complexity of propositional linear temporal logics. J. ACM 32(3) (1985) 733-749

21. Vardi, M.Y.: An automata-theoretic approach to linear temporal logic. In Moller, F., Birtwistle, G.M., eds.: Banff Higher Order Workshop. Volume 1043 of Lecture Notes in Computer Science., Springer (1995) 238-266

22. Suwimonteerabuth, D., Schwoon, S., Esparza, J.: jmoped: A java bytecode checker based on moped. In Halbwachs, N., Zuck, L.D., eds.: TACAS. Volume 3440 of Lecture Notes in Computer Science., Springer (2005) 541-545

23. Suwimonteerabuth, D., Berger, F., Schwoon, S., Esparza, J.: jmoped: A test environment for java programs. In Damm, W., Hermanns, H., eds.: CAV. Volume 4590 of Lecture Notes in Computer Science., Springer (2007) 164-167

24. Bouajjani, A., Esparza, J., Touili, T.: A generic approach to the static analysis of concurrent programs with procedures. In: POPL. (2003) 62-73

25. Bouajjani, A., Esparza, J., Schwoon, S., Strejcek, J.: Reachability analysis of multithreaded software with asynchronous communication. In Ramanujam, R., Sen, S., eds.: FSTTCS. Volume 3821 of Lecture Notes in Computer Science., Springer (2005) 348-359

26. Edmund M. Clarke, J., Grumberg, O., Peled, D.A.: Model Checking. MIT Press, Cambridge, MA (1999)

\section{Appendix}

\section{Proof of Theorem 1}

We give some preliminary definitions before the detailed proof. We define a mapping $G: \mathbb{P} \times \mathbb{Q} \times \mathbb{I} \rightarrow \mathbb{L}$ from local variables of pushdown system to memory locations of current stack in operational semantics. I contains the depth of procedure call from main to the procedure which local variable belongs to. If we have $l=G\left(p_{k}, x, i\right) \in \operatorname{dom}(\lambda)$, that means $l$ stands for local variable $x$ of procedure $p_{k}$, and $p_{k}$ is on the $i$ th position of procedure stack. Let $i_{t} \in \mathbb{I}$ be the top-most procedure of current stack. We claim a common constraint to the language semantics that currently executing procedure cannot operate on the local variables of other procedures. Thus if $l:=e(l \in \operatorname{dom}(\lambda))$ is executed in calling procedure $p_{k}$, there must be a $\left\langle p_{k}, x\right\rangle$, where $l=G\left(p_{k}, x, i_{t}\right)$ and the corresponding statement is $x:=e$. 
We inductively prove that for each derivation rule $\Phi\left(S, n_{i}, n_{j}, p, \emptyset\right)$, the transition from $\mu_{i}$ to $\mu_{j}$ is operationally sound to the change from $\left(\mu_{0}, \lambda_{0}\right)$ to $\left(\mu^{\prime}, \lambda^{\prime}\right)$ if $\left(\mu_{0}, \lambda_{0}, S\right) \downarrow$ $\left(\mu^{\prime}, \lambda^{\prime}\right)$.

\section{SKIP}

$\mu^{\prime}=\mu_{0}, \lambda^{\prime}=\lambda_{0}$. (SKIP) $\quad \rho^{\prime}=\rho_{0}, \eta^{\prime}(p)=\eta_{0}(p)$. (P-SKIP)

$\forall l \in \operatorname{dom}(\mu), \mu_{0}(l)=\rho_{0}[l] \Rightarrow \mu^{\prime}(l)=\rho^{\prime}[l]$

Since $\lambda^{\prime}=\lambda_{0}, \forall l \in \operatorname{dom}\left(\lambda^{\prime}\right) \Rightarrow l \in \operatorname{dom}\left(\lambda_{0}\right)$. If $l=G\left(p, x, i_{t}\right), \lambda_{0}(l)=\eta_{0}(p)[x]$, thus $\lambda^{\prime}(l)=\eta_{0}(p)[x]$. Since $\eta^{\prime}(p)=\eta_{0}(p)$, we have $\forall x \in \eta(p), \eta^{\prime}(p)[x]=\eta_{0}(p)[x]$. Thus $\lambda^{\prime}(l)=\eta^{\prime}(p)[x]$.

2. UPDATE

If $l_{0} \in \operatorname{dom}(\mu) .\left(l_{0} \in \rho\right)$.

$\mu^{\prime}=\mu_{0}\left[l_{0}:=v\right], \lambda^{\prime}=\lambda_{0} \cdot($ UPDATE-HEAP)

$\rho^{\prime}=\rho_{0}\left[l_{0}:=e\right], \eta^{\prime}(p)=\eta_{0}(p) .($ P-UPDATE $)$

$\forall l \in \operatorname{dom}(\mu) \backslash\left\{l_{0}\right\}, \mu^{\prime}(l)=\mu_{0}(l), \rho^{\prime}[l]=\rho_{0}[l]$, since $\mu_{0}(l)=\rho_{0}[l]$, then $\mu^{\prime}(l)=\rho^{\prime}[l]$, and since $\mu^{\prime}\left(l_{0}\right)=\rho^{\prime}\left[l_{0}\right]=v$, we have $\forall l \in \operatorname{dom}(\mu), \mu^{\prime}(l)=\rho^{\prime}[l]$.

Since $\lambda^{\prime}=\lambda_{0}, \forall l \in \operatorname{dom}\left(\lambda^{\prime}\right) \Rightarrow l \in \operatorname{dom}\left(\lambda_{0}\right)$. If $l=G\left(p, x, i_{t}\right), \lambda_{0}(l)=\eta_{0}(p)[x]$, thus $\lambda^{\prime}(l)=\eta_{0}(p)[x]$. Since $\eta^{\prime}(p)=\eta_{0}(p)$, we have $\forall x \in \eta(p), \eta^{\prime}(p)[x]=\eta_{0}(p)[x]$. Thus $\lambda^{\prime}(l)=\eta^{\prime}(p)[x]$.

If $l_{0} \in \operatorname{dom}\left(\lambda_{0}\right), \exists\left\langle p, x_{0}\right\rangle, l_{0}=G\left(p, x_{0}, i_{t}\right)$, the corresponding statement of pushdown system is $x_{0}:=e$.

$\mu^{\prime}=\mu_{0}, \lambda^{\prime}=\lambda_{0}\left[l_{0}:=v\right]$. (UPDATE-STACK)

$\rho^{\prime}=\rho_{0}, \eta^{\prime}(p)=\eta_{0}(p)\left[x_{0}:=e\right]$. (P-UPDATE)

$\forall l \in \operatorname{dom}(\mu), \mu^{\prime}(l)=\mu(l), \rho^{\prime}[l]=\rho_{0}[l]$, since $\mu_{0}(l)=\rho_{0}[l]$, then $\mu^{\prime}(l)=\rho^{\prime}[l]$.

$\forall l \in \operatorname{dom}\left(\lambda^{\prime}\right) \backslash\left\{l_{0}\right\}, \lambda^{\prime}(l)=\lambda_{0}(l)$, if $l=G\left(p, x, i_{t}\right), \lambda_{0}(l)=\eta_{0}(p)[x] \wedge x \neq x_{0}$, thus we have $\eta^{\prime}(p)[x]=\eta_{0}(p)[x], \lambda^{\prime}(l)=\lambda_{0}(l)=\eta_{0}(p)[x]=\eta^{\prime}(p)[x]$. Also we have $\lambda^{\prime}\left(l_{0}\right)=\eta^{\prime}(p)\left[x_{0}\right]=v$. Therefore $\forall l \in \operatorname{dom}\left(\lambda^{\prime}\right)$, if $l=G\left(p, x, i_{t}\right), \lambda^{\prime}(l)=\eta^{\prime}(p)[x]$.

3. SEQ

$$
\begin{aligned}
& \left(\begin{array}{c}
\left(\begin{array}{l}
\forall l \in \operatorname{dom}\left(\mu_{1}\right), \mu_{1}(l)=\rho_{1}[l] \wedge \\
\forall l \in \operatorname{dom}\left(\lambda_{1}\right), \text { if } \exists\langle p, x\rangle, l=G\left(p, x, i_{t}\right), \text { then } \lambda_{1}(l)=\eta_{1}(p)[x]
\end{array}\right) \Rightarrow \\
\left(\begin{array}{l}
\forall l \in \operatorname{dom}\left(\mu_{1}\right), \mu_{1}^{\prime}(l)=\rho_{1}^{\prime}[l] \wedge \\
\forall l \in \operatorname{dom}\left(\lambda_{1}^{\prime}\right), \text { if } \exists\langle p, x\rangle, l=G\left(p, x, i_{t}\right), \text { then } \lambda_{1}^{\prime}(l)=\eta_{1}^{\prime}(p)[x]
\end{array}\right)
\end{array}\right) \\
& \left(\left(\begin{array}{l}
\forall l \in \operatorname{dom}\left(\mu_{2}\right), \mu_{2}(l)=\rho_{2}[l] \wedge \\
\forall l \in \operatorname{dom}\left(\lambda_{2}\right), \text { if } \exists\langle p, x\rangle, l=G\left(p, x, i_{t}\right) \text {, then } \lambda_{2}(l)=\eta_{2}(p)[x]
\end{array}\right) \Rightarrow\right. \\
& \left(\forall l \in \operatorname{dom}\left(\mu_{2}\right), \mu_{2}^{\prime}(l)=\rho_{2}^{\prime}[l] \wedge\right. \\
& \left(\forall l \in \operatorname{dom}\left(\lambda_{2}^{\prime}\right) \text {, if } \exists\langle p, x\rangle, l=G\left(p, x, i_{t}\right) \text {, then } \lambda_{2}^{\prime}(l)=\eta_{2}^{\prime}(p)[x]\right) \\
& \left(\begin{array}{l}
\forall l \in \operatorname{dom}\left(\mu_{0}\right), \mu_{0}(l)=\rho_{0}[l] \wedge \\
\forall l \in \operatorname{dom}\left(\lambda_{0}\right), \text { if } \exists\langle p, x\rangle, l=G\left(p, x, i_{t}\right) \text {, then } \lambda_{0}(l)=\eta_{0}(p)[x]
\end{array}\right) \wedge \\
& \mu_{1}=\mu_{0} \wedge \lambda_{1}=\lambda_{0} \wedge \rho_{1}=\rho_{0} \wedge \eta_{1}(p)=\eta_{0}(p) \wedge(\mathrm{I}) \Rightarrow \\
& \left(\begin{array}{l}
\forall l \in \operatorname{dom}\left(\mu_{1}\right), \mu_{1}^{\prime}(l)=\rho_{1}^{\prime}[l] \wedge \\
\forall l \in \operatorname{dom}\left(\lambda_{1}^{\prime}\right), \text { if } \exists\langle p, x\rangle, l=G\left(p, x, i_{t}\right), \text { then } \lambda_{1}^{\prime}(l)=\eta_{1}^{\prime}(p)[x]
\end{array}\right) \\
& \mu_{2}=\mu_{1}^{\prime} \wedge \lambda_{2}=\lambda_{1}^{\prime} \wedge \rho_{2}=\rho_{1}^{\prime} \wedge \eta_{2}(p)=\eta_{1}^{\prime}(p) \wedge(\mathrm{III}) \Rightarrow \\
& \left(\begin{array}{l}
\forall l \in \operatorname{dom}\left(\mu_{2}\right), \mu_{2}^{\prime}(l)=\rho_{2}^{\prime}[l] \wedge \\
\forall l \in \operatorname{dom}\left(\lambda_{2}^{\prime}\right), \text { if } \exists\langle p, x\rangle, l=G\left(p, x, i_{t}\right), \text { then } \lambda_{2}^{\prime}(l)=\eta_{2}^{\prime}(p)[x]
\end{array}\right) \\
& \mu^{\prime}=\mu_{2}^{\prime} \wedge \lambda^{\prime}=\lambda_{2}^{\prime} \wedge \rho^{\prime}=\rho_{2}^{\prime} \wedge \eta^{\prime}(p)=\eta_{2}^{\prime}(p) \wedge(\mathrm{IV}) \Rightarrow \\
& \left(\begin{array}{l}
\forall l \in \operatorname{dom}\left(\mu^{\prime}\right), \mu^{\prime}(l)=\rho^{\prime}[l] \wedge \\
\forall l \in \operatorname{dom}\left(\lambda^{\prime}\right), \text { if } \exists\langle p, x\rangle, l=G\left(p, x, i_{t}\right), \text { then } \lambda^{\prime}(l)=\eta^{\prime}(p)[x]
\end{array}\right) .
\end{aligned}
$$

4. CONDITIONAL-BRANCH

If $\left(\mu_{0}, \lambda_{0}, e\right) \downarrow$ true, the branch conditions in $R$ show that the transition from $n_{i}$ to $n_{j}$ is executed by $\left\{\left\langle\left(\rho_{i}\right)\left(n_{i}, \eta_{i}(p)\right)\right\rangle \hookrightarrow\left\langle\left(\rho_{i}\right)\left(n_{k}, \eta_{i}(p)\right)\right\rangle \mid R \cup\{e\}\right\}$ and the rules 
generated by $\Phi\left(S_{1}, n_{k}, n_{j}, p, R \cup\{e\}\right)$. We have $\rho_{k}=\rho_{0} \wedge \eta_{k}(p)=\eta_{0}(p)$,

$\left(\begin{array}{l}\forall l \in \operatorname{dom}\left(\mu_{0}\right), \mu_{0}(l)=\rho_{0}[l]=\rho_{k}[l] \wedge \\ \forall l \in \operatorname{dom}\left(\lambda_{0}\right), \text { if } \exists\langle p, x\rangle, l=G\left(p, x, i_{t}\right), \text { then } \lambda_{0}(l)=\eta_{0}(p)[x]=\eta_{k}(p)[x]\end{array}\right)$

We can inductively get

$\left(\begin{array}{l}\forall l \in \operatorname{dom}\left(\mu^{\prime}\right), \mu^{\prime}(l)=\rho^{\prime}[l] \wedge \\ \forall l \in \operatorname{dom}\left(\lambda^{\prime}\right), \text { if } \exists\langle p, x\rangle, l=G\left(p, x, i_{t}\right), \text { then } \lambda^{\prime}(l)=\eta^{\prime}(p)[x]\end{array}\right)$

If $\left(\mu_{0}, \lambda_{0}, e\right) \downarrow$ false, the proof is similar.

\section{LOOP}

If $\left(\mu_{0}, \lambda_{0}, e\right) \downarrow$ false, $\mu^{\prime}=\mu_{0}, \lambda^{\prime}=\lambda_{0}$. (WHILE-F)

From the branch condition in $R$ of (P-LOOP) we can see the transition is executed by pushdown rule $\left\{\left\langle\left(\rho_{i}\right)\left(n_{i}, \eta_{i}(p)\right)\right\rangle \hookrightarrow\left\langle\left(\rho_{i}\right)\left(n_{j}, \eta_{i}(p)\right)\right\rangle \mid R \cup\{! e\}\right\}$. Thus $\rho^{\prime}=\rho_{0}, \eta^{\prime}(p)=\eta_{0}(p)$.

$\forall l \in \operatorname{dom}\left(\mu_{0}\right), \mu_{0}(l)=\rho_{0}[l] \Rightarrow \mu^{\prime}(l)=\rho^{\prime}[l]$

Since $\lambda^{\prime}=\lambda_{0}, \forall l \in \operatorname{dom}\left(\lambda^{\prime}\right) \Rightarrow l \in \operatorname{dom}\left(\lambda_{0}\right)$. If $l=G\left(p, x, i_{t}\right), \lambda_{0}(l)=\eta_{0}(p)[x]$, thus $\lambda^{\prime}(l)=\eta_{0}(p)[x]$. Since $\eta^{\prime}(p)=\eta_{0}(p)$, then $\forall x \in \eta(p), \eta^{\prime}(p)[x]=\eta_{0}(p)[x]$. Thus $\lambda^{\prime}(l)=\eta^{\prime}(p)[x]$.

If $\left(\mu_{0}, \lambda_{0}, e\right) \downarrow$ true, we need to prove the execution of pushdown system before the next time evaluation of $e$ is sound to operational semantics.

$\rho_{q}=\rho_{0}, \eta_{q}(p)=\eta_{0}(p)$.(P-LOOP)

$\left(\forall l \in \operatorname{dom}\left(\mu_{0}\right), \mu_{0}(l)=\rho_{0}[l]=\rho_{q}[l] \wedge\right.$

$\forall l \in \operatorname{dom}\left(\lambda_{0}\right)$, if $\exists\langle p, x\rangle, l=G\left(p, x, i_{t}\right)$, then $\left.\lambda_{0}(l)=\eta_{0}(p)[x]=\eta_{q}(p)[x]\right)$

$\Rightarrow\left(\begin{array}{l}\forall l \in \operatorname{dom}\left(\mu^{\prime}\right), \mu^{\prime}(l)=\rho_{i}^{\prime}[l] \wedge \\ \forall l \in \operatorname{dom}\left(\lambda^{\prime}\right), \text { if } \exists\langle p, x\rangle, l=G\left(p, x, i_{t}\right), \text { then } \lambda^{\prime}(l)=\eta_{i}^{\prime}(p)[x]\end{array}\right)$.

\section{BINDVAR}

$\left(\begin{array}{l}\forall l \in \operatorname{dom}\left(\mu_{0}\right), \mu_{0}(l)=\rho_{0}[l] \wedge \\ \forall l \in \operatorname{dom}\left(\lambda_{0}\right), \text { if } \exists\langle p, x\rangle, l=G\left(p, x, i_{t}\right), \text { then } \lambda_{0}(l)=\eta_{0}(p)[x]\end{array}\right)$

$\left(\begin{array}{c}\left(\begin{array}{l}\forall l \in \operatorname{dom}\left(\mu_{1}\right), \mu_{1}(l)=\rho_{1}[l] \wedge \\ \forall l \in \operatorname{dom}\left(\lambda_{1}\right), \text { if } \exists\langle p, x\rangle, l=G\left(p, x, i_{t}\right), \text { then } \lambda_{1}(l)=\eta_{1}(p)[x]\end{array}\right) \Rightarrow \\ \left(\begin{array}{l}\forall l \in \operatorname{dom}\left(\mu_{2}\right), \mu_{2}(l)=\rho_{2}[l] \wedge \\ \forall l \in \operatorname{dom}\left(\lambda_{2}\right), \text { if } \exists\langle p, x\rangle, l=G\left(p, x, i_{t}\right), \text { then } \lambda_{2}(l)=\eta_{2}(p)[x]\end{array}\right)\end{array}\right)$

$\mu_{1}=\mu_{0}, \lambda_{1}=\lambda_{0} \uplus\left[l_{0}:=v\right]\left(l_{0} \notin \operatorname{dom}\left(\mu_{0}\right) \wedge l_{0} \notin \operatorname{dom}\left(\lambda_{0}\right)\right)$. (BINDVAR)

$\rho_{1}=\rho_{0}, \eta_{1}(p)=\eta_{0}(p)\left[x_{0}:=e\right]$. (P-BINDVAR)

Therefore, $\forall l \in \operatorname{dom}(\mu), \mu_{1}(l)=\rho_{1}[l]$.

$\forall l \in \operatorname{dom}\left(\lambda_{1}\right) \backslash\left\{l_{0}\right\}, l \in \operatorname{dom}\left(\lambda_{0}\right) \wedge \lambda_{0}(l)=\lambda_{1}(l)$, we have, if $\exists\langle p, x\rangle, l=G\left(p, x, i_{t}\right)$, then $\lambda_{0}(l)=\eta_{0}(p)[x] \wedge x \neq x_{0}$, thus $\eta_{1}(p)[x]=\eta_{0}(p)[x]$.

Therefore $\forall l \in \operatorname{dom}\left(\lambda_{1}\right) \backslash\left\{l_{0}\right\}$, if $\exists\langle p, x\rangle, l=G\left(p, x, i_{t}\right), \lambda_{1}(l)=\eta_{1}(p)[x]$. And since $\lambda_{1}\left(l_{0}\right)=\eta_{1}(p)\left[x_{0}\right]=v$, we have $\forall l \in \operatorname{dom}\left(\lambda_{1}\right)$, if $\exists\langle p, x\rangle, l=G\left(p, x, i_{t}\right)$, then $\lambda_{1}(l)=$ $\eta_{1}(p)[x]$.

(IV)

From (III) (IV)(II) we have

$\left(\forall l \in \operatorname{dom}\left(\mu_{2}\right), \mu_{2}(l)=\rho_{2}[l] \wedge\right.$

$\forall l \in \operatorname{dom}\left(\lambda_{2}\right)$, if $\exists\langle p, x\rangle, l=G\left(p, x, i_{t}\right)$, then $\left.\lambda_{2}(l)=\eta_{2}(p)[x]\right)$

$\mu^{\prime}=\mu_{2}, \lambda^{\prime}=\lambda_{2} \backslash\left\{l_{0}\right\}$. (BINDVAR) $\quad \rho^{\prime}=\rho_{2}, \eta^{\prime}(p)=\eta_{2}(p)$. (P-BINDVAR)

Therefore, $\left(\begin{array}{l}\forall l \in \operatorname{dom}\left(\mu^{\prime}\right), \mu^{\prime}(l)=\rho^{\prime}[l] \wedge \\ \forall l \in \operatorname{dom}\left(\lambda^{\prime}\right), \text { if } \exists\langle p, x\rangle, l=G\left(p, x, i_{t}\right), \text { then } \lambda^{\prime}(l)=\eta^{\prime}(p)[x]\end{array}\right)$

7. PROC

$\left(\forall l \in \operatorname{dom}\left(\mu_{0}\right), \mu_{0}(l)=\rho_{0}[l] \wedge\right.$

$\forall l \in \operatorname{dom}\left(\lambda_{0}\right)$, if $\exists\langle p, x\rangle, l=G\left(p, x, i_{t}\right)$, then $\left.\lambda_{0}(l)=\eta_{0}(p)[x]\right)$ 
$\left(\begin{array}{c}\left(\begin{array}{l}\forall l \in \operatorname{dom}\left(\mu_{1}\right), \mu_{1}(l)=\rho_{1}[l] \wedge \\ \forall l \in \operatorname{dom}\left(\lambda_{1}\right), \text { if } \exists\left\langle p^{\prime}, x\right\rangle, l=G\left(p^{\prime}, x, i_{t}\right), \text { then } \lambda_{1}(l)=\eta_{1}\left(p^{\prime}\right)[x]\end{array}\right) \Rightarrow \\ \left(\begin{array}{l}\forall l \in \operatorname{dom}\left(\mu_{2}\right), \mu_{2}(l)=\rho_{2}[l] \wedge \\ \forall l \in \operatorname{dom}\left(\lambda_{2}\right), \text { if } \exists\left\langle p^{\prime}, x\right\rangle, l=G\left(p^{\prime}, x, i_{t}\right), \text { then } \lambda_{2}(l)=\eta_{2}\left(p^{\prime}\right)[x]\end{array}\right)\end{array}\right)$

$\mu_{1}=\mu_{0}, \lambda_{1}=\lambda_{0} \uplus\left[l^{\prime}:=v\right]\left(l^{\prime} \notin \operatorname{dom}\left(\mu_{0}\right) \wedge l^{\prime} \notin \operatorname{dom}\left(\lambda_{0}\right)\right)$. (CALL)

$\rho_{1}=\rho_{0}$. Therefore $\forall l \in \operatorname{dom}\left(\mu_{1}\right), \mu_{1}(l)=\rho_{1}[l]$.

$\forall l \in \operatorname{dom}\left(\lambda_{1}\right) \backslash\left\{l^{\prime}\right\}, l \in \operatorname{dom}\left(\lambda_{0}\right), \neg \exists\left\langle p^{\prime}, x\right\rangle\left(l=G\left(p^{\prime}, x, i_{t}\right)\right)$.

$l^{\prime}=G\left(p^{\prime}, x_{1}, i_{t}\right) \wedge \lambda_{1}\left(l^{\prime}\right)=\eta_{1}\left(p^{\prime}\right)\left[x_{1}\right]=v$.

Therefore, $\forall l \in \operatorname{dom}\left(\lambda_{1}\right)$, if $\exists\left\langle p^{\prime}, x\right\rangle, l=G\left(p^{\prime}, x, i_{t}\right)$, then $\lambda_{1}(l)=\eta_{1}\left(p^{\prime}\right)[x]$.

From (III)(IV)(II) we have

$\left(\forall l \in \operatorname{dom}\left(\mu_{2}\right), \mu_{2}(l)=\rho_{2}[l] \wedge\right.$

$\forall l \in \operatorname{dom}\left(\lambda_{2}\right)$, if $\exists\left\langle p^{\prime}, x\right\rangle, l=G\left(p^{\prime}, x, i_{t}\right)$, then $\left.\lambda_{2}(l)=\eta_{2}\left(p^{\prime}\right)[x]\right)$

$\mu^{\prime}=\mu_{2}, \lambda^{\prime}=\lambda_{2} \backslash\left\{l^{\prime}\right\}$. (CALL) $\quad \rho^{\prime}=\rho_{2}, \eta^{\prime}(p)=\eta_{0}(p)$. (P-PROC)

Therefore, $\forall l \in \operatorname{dom}(\mu), \mu^{\prime}(l)=\rho^{\prime}[l]$.

Procedure $p$ is on top of procedure stack, so $\operatorname{dom}\left(\lambda_{0}\right)=\operatorname{dom}\left(\lambda^{\prime}\right)$. Since procedure $p^{\prime}$ cannot modify local variables in $\lambda^{\prime}$, we have $\forall l \in \operatorname{dom}\left(\lambda^{\prime}\right), \lambda^{\prime}(l)=\lambda_{0}(l)$. Since $\eta^{\prime}(p)=\eta_{0}(p)$, we have $\forall l \in \operatorname{dom}\left(\lambda^{\prime}\right)$, if $\exists\langle p, x\rangle, l=G\left(p, x, i_{t}\right)$, then $\lambda^{\prime}(l)=\eta^{\prime}(p)[x]$. 